\section{Real stenosis in the vein graft ostium? The aortic wall protrusion may mislead you}

The aorto-ostial junction is a unique segment of the coronary arterial tree. The angle of take-off of the proximal coronary vessels and the coaxial positioning and disengagement of the guide may result in angiographic ambiguity or misinterpretation of true ostial disease. The aortic wall protrusion adjacent to anastomosis after coronary artery bypass graft is extremely rare. A 68-year-old man who had undergone CABG 6 months prior was referred to our institution for the treatment of chest pain. Coronary angiography revealed a slender, slit-like ostial
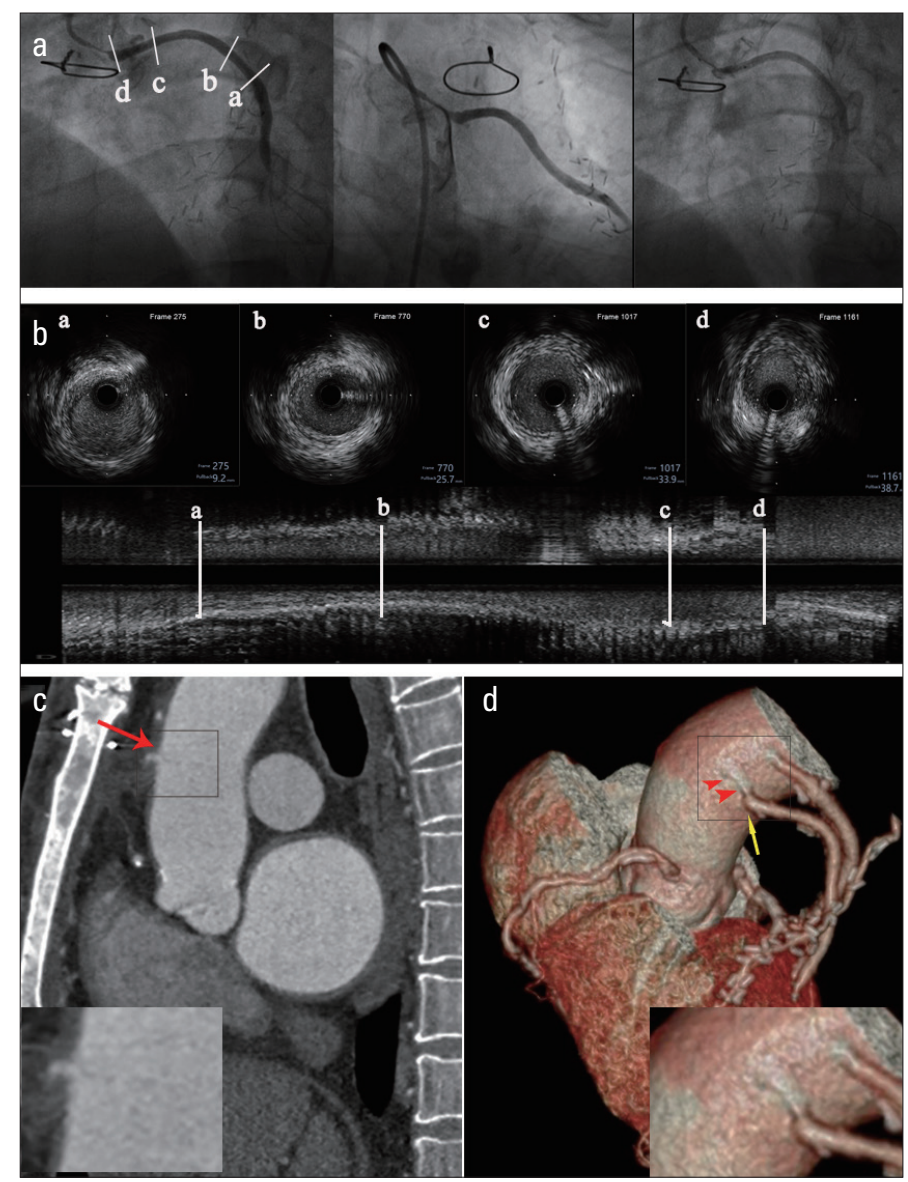

Figure 1. (a) Initial coronary angiogram of the vein graft demonstrating a slender, slit-like stenosis in the ostium; (b) Intravascular ultrasound with cross-sectional and longitudinal images; (c, d) Contrast-enhanced computed tomography showing wall protrusion adjacent to the aortic anastomosis. Guiding tip slid into the wall protrusion (red arrow) adjacent to anastomosis (yellow arrow) stenosis of the saphenous vein graft on the diagonal branch (Fig. 1 a and Videos 1 and 2). Graft atherosclerosis was considered to be responsible for lumen loss, and implantation of a drug-eluting stent was planned to treat this lesion. Balloon angioplasty was performed, and intravascular ultrasound was subsequently performed to better characterize the plaque. However, the intravascular ultrasound revealed significant wall thickening without lipid-rich plaque throughout the vein graft and no severe stenosis in the ostium (Fig. 1b and Video 3). The procedure was suspended owing to the ambiguous lesion at the aortic anastomosis. A subsequent contrast-enhanced computed tomography scan revealed a wall protrusion near the aortic anastomosis (Fig. 1c, 1d). This protrusion was attributed to a surgical suture in the weakened and degenerative aortic wall near the anastomosis after consultation with the patient's cardiac surgeon. The guiding tip may have slid into this wall protrusion during coronary angiography, and the slit-like protrusion was mistaken for graft ostium stenosis. It would be dangerous if a stent was implanted at this lesion. The patient was discharged after receiving the optimal medical therapy.

Institutional and financial support: This work was supported by a grant from the Tianjin Municipal Health Bureau (CN) (Grant number: MS20016, funder: Yue-Cheng Hu).

Informed consent: Written consent was obtained from the patient.

Video 1, 2. Initial coronary angiogram of the vein graft demonstrating a slender, slit-like stenosis in the vein graft ostium

Video 3. Intravascular ultrasound demonstrating significant wall thickening without lipid-rich plaque throughout the vein graft and no severe stenosis in the ostium

\section{Chun-Wei Liu1 ${ }^{* *}(\mathbb{D}$, Zhi-Gang Guo* (D), Yue-Cheng Hu** (D), Hong-Liang Cong** (D)}

\section{Tianjin Medical University; Tianjin-China \\ Departments of *Cardiac Surgery, and **Cardiology, Tianjin Chest Hospital; Tianjin-China}

Address for Correspondence: Hong-Liang Cong, MD,

Department of Cardiology, Tianjin Medical University, Tianjin Chest Hospital; Tianjin-China

Phone: +8613702012871

E-mail: conghongliang2871@163.com

(C) Copyright 2021 by Turkish Society of Cardiology -

Available online at

www.anatoljcardiol.com

DOI:10.5152/AnatolJCardiol.2021.435 